\title{
Olive oil consumption and 10-year (2002-2012) cardiovascular disease incidence: the ATTICA Study
}

Georgia-Maria Kouli ${ }^{1}$, MSc, Demosthenes B. Panagiotakos ${ }^{1,5}$, PhD, Ioannis Kyrou $^{1,2,3,4}$, MD, PhD, Emanuela Magriplis ${ }^{1}$, PhD, Ekavi N Georgousopoulou ${ }^{1,5}$, $\mathrm{PhD}$, Christina Chrysohoou ${ }^{6}, \mathrm{MD}, \mathrm{PhD}$, Constantine Tsigos ${ }^{1}, \mathrm{MD}, \mathrm{PhD}$, Dimitrios Tousoulis ${ }^{6}, \mathrm{MD}, \mathrm{PhD}$, Christos Pitsavos ${ }^{6}, \mathrm{MD}, \mathrm{PhD}$.

${ }^{1}$ Department of Nutrition and Dietetics, School of Health Science and Education Harokopio University, Athens, Greece; ${ }^{2}$ Aston Medical Research Institute, Aston Medical School, Aston University, B4 7ET, Birmingham, UK; ${ }^{3}$ WISDEM, University Hospital Coventry and Warwickshire NHS Trust, CV2 2DX, Coventry, UK; ${ }^{4}$ Translational \& Experimental Medicine, Division of Biomedical Sciences, Warwick Medical School, University of Warwick, CV4 7AL, Coventry, UK; ${ }^{5}$ Faculty of Health, University of Canberra, Canberra, Australia; ${ }^{6}$ First Cardiology Clinic, School of Medicine, University of Athens, Greece

\section{Acknowledgements}

The authors would like to thank the ATTICA study group of investigators: Yannis Skoumas, Natasa Katinioti, Labros Papadimitriou, Constantina Masoura, Spiros Vellas, Yannis Lentzas, Manolis Kambaxis, Konstanitna Paliou, Vassiliki Metaxa, Agathi Ntzouvani, Dimitris Mpougatas, Nikolaos Skourlis, Christina Papanikolaou, Aikaterini Kalogeropoulou, Evangelia Pitaraki, Alexandros Laskaris, Mihail Hatzigeorgiou and Athanasios Grekas, Eleni Kokkou for either assistance in the initial physical examination and follow-up evaluation, Efi Tsetsekou for her assistance in psychological evaluation and follow-up evaluation, as well as laboratory team: Carmen Vassiliadou and George Dedousis (genetic analysis), Marina ToutouzaGiotsa, Constantina Tselika and Sia Poulopouloou (biochemical analysis) and Maria Toutouza for the database management.

\section{Corresponding author}

Prof Demosthenes B Panagiotakos

Harokopio University, 17671 Athens, Greece

Tel. +30 210-9549332

Email: dbpanag@hua.gr 


\section{Abstract}

Purpose: Olive oil, being rich in mono-unsaturated fatty acids and anti-inflammatory compounds, may have protective effects against cardiovascular disease (CVD). The aim of the present work was to examine the association of olive oil consumption with the 10-year CVD incidence in adults without pre-existing CVD. Methods: The ATTICA Study is a prospective, population-based study conducted in the greater metropolitan area of Athens (Attica, Greece). During 2001-2002, 3042 CVD-free adults (1514 men and 1528 women) were voluntarily recruited to the ATTICA study. Among various dietary habits, consumption of olive oil and other fats/oils was assessed at baseline; participants were classified into three groups (no use; mixed use; and exclusive use of olive oil). In 2011-2012, the 10-year study follow-up was performed, recording the fatal/non-fatal CVD incidence in 2020 participants (mean follow-up duration: 8.41 years). Results: After controlling for various covariates, an inverse association between exclusive olive oil use and the risk of developing CVD was observed (relative risk 0.07, 95\%CI: 0.01-0.66) compared to those not consuming olive oil. Further adjustment for fibrinogen plasma levels (among various inflammatory markers) showed a significant mediation effect on the previous association. Conclusions: These findings support exclusive olive oil consumption, a key component of the Mediterranean diet, for the primary CVD prevention, in adults without pre-existing disease. Circulating fibrinogen levels appear to play a mediating role in this relationship.

Keywords: olive oil, cardiovascular disease, nutrition, inflammation, fibrinogen 


\section{Introduction}

Non-communicable diseases account for $70 \%$ of all deaths globally, with cardiovascular disease (CVD) constituting the most common cause (3.9 million CVDrelated deaths in Europe, accounting for $45 \%$ of all deaths) [1]. Notably, mortality rates from ischaemic heart disease and stroke tend to be generally higher in Central and Eastern Europe than in Western and Southern Europe (e.g. in France, Spain, Greece, Italy and Portugal) [1, 2], contributing to the trends for longer life expectancy in these countries. Among the behavioral CVD risk factors, dietary factors are considered to have the greatest impact on CVD mortality/morbidity [1]. Indeed, the Mediterranean diet has been proposed as one of the major protective factors responsible for these geographical disparities in CVD mortality trends [3, 4].

The typical Mediterranean diet represents the traditional dietary pattern that prevailed in olive-growing areas of the Mediterranean region, such as the Greek island of Crete and southern Italy, in the late 1950s and early 1960s [5]. As such, olive oil, being the main source of culinary fat in these countries/regions $[6,7]$, is regarded as a key component of the traditional Mediterranean diet. In recent years, several health benefits have been credited to olive oil (virgin or refined) consumption and its nutritional value [8]. These are mainly attributed to its high content of monounsaturated fatty acids (MUFA), antioxidants and anti-inflammatory compounds, which may exert beneficial effects against the development and progression of diseases associated with chronic low-grade inflammation, including CVD where chronic inflammation of the vascular endothelium is linked to CVD events [8].

Compiling epidemiological data suggest that olive oil intake is inversely associated with CVD [9-12]. In the Spanish branch of the European Prospective Investigation into Cancer and Nutrition (EPIC) study, the total olive oil intake was associated with decreased risk of coronary heart disease (CHD) and lower all-cause 
and CVD mortality [10]. Similarly, the Prevention through Mediterranean Diet (PREDIMED) randomized clinical trial showed that a Mediterranean diet supplemented with olive oil reduces the incidence of CVD episodes and mortality in high CVD risk patients [11]. Moreover, in a case-control study conducted in Greece exclusive use of olive oil was also found to have a significant protective effect against acute coronary syndromes [12].

Despite the increasing evidence supporting the protective effects of olive oil consumption in relation to CVD and chronic subclinical inflammation [8], limited epidemiological data exist from the general population on the relationship between olive oil intake and long-term CVD risk. Therefore, the aim of the current work was to prospectively examine the association of olive oil consumption with the 10 -year CVD incidence in the ATTICA study cohort of adults without pre-existing CVD.

\section{Materials and Methods}

\subsection{Sampling procedure at baseline}

The ATTICA Study is a prospective, population-based study conducted in the greater metropolitan area of Athens (Attica region, Greece). The study baseline examination was performed during 2001-2002, as previously described [13]. Briefly, the study was designed to enroll only one participant per household through a random, multistage process based on the age/sex distribution of the general population of the Attica region (2001 National Census). A total of 3042 free-living, Caucasian adults [1514 men (age: 18-87 years); 1528 women (age: 18-89 years)] consented to participate and were assessed by trained personnel, as per study protocol [13]. The study's physicians performed exclusion of individuals with pre-existing CVD at baseline through a detailed clinical evaluation. 


\subsection{Baseline measurements}

The baseline evaluation included data on demographic characteristics (e.g., age, sex, education), personal/family medical history (e.g. hypercholesterolemia, hypertension and diabetes), as well as dietary and other lifestyle habits (e.g. smoking status and physical activity) [13]. In the context of the present study, smokers were defined as those who smoked at least one cigarette per day or had stopped smoking during the preceding year (the rest of the participants were defined as non-smokers), and education was measured in years of school. Dietary habits were assessed based on a validated semi-quantitative food-frequency questionnaire (FFQ) [14], namely the EPIC-Greek questionnaire, which was kindly provided by the Unit of Nutrition of Athens Medical School. Moreover, the MedDietScore was also applied to assess the adherence to the Mediterranean diet (range 0-55; higher score values indicating greater adherence to the Mediterranean diet), as previously described [15]. In addition, study participants were asked to report the average intake of several foods and liquids consumed during the previous 12 months, and data on the total energy intake (kcal/day), ethanol intake (g/d), and percent (\%) energy intake from fat were calculated based on food composition tables. Moreover, the intake of MUFA, saturated fatty acids (SFA), and trans-fatty acids (TFA), as well as the MUFA/SFA intake ratio and omega-3 fatty acids, were also calculated based on the information retrieved through the FFQ and relevant food composition tables. To evaluate the baseline physical activity status of study participants the International Physical Activity Questionnaire was used (IPAQ) [16], as an index of weekly energy expenditure using frequency (times per week), duration (in minutes per time) and intensity of sports or other habits related to physical activity (in expended calories per 
time). Accordingly, participants who did not report any physical activities were defined as physically inactive.

Anthropometric measurements were performed for all participants at baseline and the body mass index (BMI) was calculated as weight (in Kg) divided by standing height (in square meters, $m^{2}$ ). The waist-to-hip ratio (WHR) was also calculated for each participant based on the hip and waist circumference measured (to the nearest $0.5 \mathrm{~cm}$ ) using an inelastic measuring tape at the iliac crest and in the middle between the lowest rib and the iliac crest, respectively.

Arterial blood pressure (mean of 3 recordings) was also measured at the end of the baseline physical examination with the study participant in a sitting position after resting for at least 30 minutes. Participants with average blood pressure levels greater or equal to 140/90 mmHg (or on antihypertensive treatment) were classified as having hypertension.

Baseline blood samples were also collected from each participant at 8-10 am after 10-12 hours of overnight fasting and alcohol abstinence. From these samples, blood lipids (total cholesterol, HDL-cholesterol and triglycerides) were measured using a chromatographic enzymic method in a Technicon automatic analyser RA1000 (Dade Behring, Marburg, Germany). Hypercholesterolemia was defined as total cholesterol levels greater than $200 \mathrm{mg} / \mathrm{dl}$ (or treatment with lipid-lowering agents). Blood glucose levels (mg/dl) were measured with a Beckman Glucose Analyzer (Beckman Instruments, Fullerton, CA, USA), and participants with fasting blood glucose levels greater than $125 \mathrm{mg} / \mathrm{dl}$ were classified as having diabetes according to the American Diabetes Association diagnostic criteria [17]. In addition, measurements of selected circulating pro-inflammatory biomarkers were also performed, including C-reactive protein (CRP), fibrinogen, and interleukin-6 (IL-6), as well as 
homocysteine and plasma oxidized LDL-cholesterol (ox-LDL; as an oxidative stress biomarker). High sensitivity CRP was assayed by particle-enhanced immunonephelometry (N Latex, Dade-Behring Marburg GmbH, Marburg, Germany) and participants with CRP levels above $10 \mathrm{mg} / \mathrm{L}$ were excluded from the analyses.

In the context of this work, the participants were also classified according to the presence/absence of metabolic syndrome (MetS), using the criteria of the NCEP ATP III (revised 2005) MetS definition [18].

\subsection{Olive oil consumption at baseline}

All participants provided detailed information on the consumption of olive oil, vegetable seed oils, butter and margarines by reporting the use of various oils in daily cooking and preparation of meals (e.g. addition to salads, dressings, etc.). Based on the reported baseline olive oil consumption, study participants were divided to those reporting daily olive oil use/consumption and those reporting no olive oil intake at all, and were also further sub-grouped into three categories, namely to no olive oil use group (consumption of other dietary fats/oils except for olive oil), mixed use group (combined use of olive oil and other dietary fats/oils) and exclusive olive oil use group (consumption/use of olive oil only).

\subsection{Follow-up examination (2011-2012)}

During 2011-12, the 10-year follow-up of the ATTICA Study was performed by trained study investigators (mean follow-up duration: 8.41 years), as previously described [19]. In brief, 2583 of the 3042 initially enrolled participants were reevaluated for this follow-up [85\% participation rate; mean age at baseline (years \pm standard deviation): $45 \pm 14$ and $46 \pm 14$ years for women and men, respectively, with no difference to the overall study sample]. Detailed evaluation of the medical records of all these re-evaluated participants $(n=2583)$ was performed, focusing on their 10 - 
year CVD status, and data (with WHO-ICD coding) were collected for: (a) vital status

(death from any cause or due to CVD); (b) development of CVD, including myocardial infarction, angina pectoris, other identified forms of ischemia, heart failure of different types, chronic arrhythmias and stroke [19]. Regarding the CVD status at the 10-year follow-up, clinically accurate and complete data were obtained from 2020 study participants (Table 1). For the aims of the present study in order to explore the association of olive oil consumption with long-term CVD risk, herein we present our data in relation to the 10-year incidence of fatal/non-fatal CVD for these 2020 study participants.

\subsection{Bioethics}

The study was approved by the Bioethics Committee of Athens Medical School and was carried out in accordance to the Declaration of Helsinki (1989) of the World Medical Association. Prior to any study-related procedures, each participant was informed on the aims and protocol of the study and provided written signed consent.

\subsection{Statistical analysis}

Crude, non-fatal and fatal incidence rates of combined CVD (i.e., coronary heart disease or stroke) were calculated as the ratio of new cases to the number of study participants in the 10-year follow-up. Continuous variables were tested for normality through P-P plots and are presented as mean values \pm standard deviation (SD) or as median if not normally distributed, whilst categorical variables are presented as frequencies. Associations between categorical variables were tested using the chi-square test, while between continuous variables using the Pearson $r$ or Spearman's rho coefficients for the normally and skewed variables, respectively. Comparisons of mean values of normally distributed variables by olive oil 
use/consumption were performed using analysis of variance (ANOVA), and post-hoc analyses using the Bonferroni rule were performed to account for the inflation of the probability of type-I error. Comparisons between mean values of normally distributed variables between those who developed a CVD event and the rest of the participants who remained CVD-free were performed using Student's t-test, after controlling for equality of variances using the Levene's test. Comparisons of continuous variables that did not follow a normal distribution were performed using the non-parametric Utest proposed by Mann and Whitney and the Kruskal-Wallis H-test. The hazard ratios (HR) and the corresponding 95\% confidence intervals (CI) of developing a CVD event during the 10-year period according to the participants' baseline characteristics were estimated using Cox proportional hazards models. The time to CVD event was recorded on an annual basis. Multicolinearity between independent variables was tested using the Correlation Matrix of the models' estimates. Sobel's test was applied to test for a potential mediating effect of various biomarkers on the tested association. All reported $p$-values are based on two-sided tests. The Statistical Package for Social Sciences (SPSS) version 21 (SPSS Inc., Chicago, IL, USA) software was used for all statistical analyses.

\section{Results}

\subsection{Baseline characteristics of study participants by olive oil consumption status}

Based on the baseline dietary reports, $89.5 \%$ of the 2020 participants reported daily olive oil consumption (mixed with other additive fats or exclusive, while the rest (10.5\%) reported no olive oil use/consumption; particularly, exclusive olive oil use in daily cooking was reported by $1.1 \%$ of the participants. Table 1 presents the demographic, clinical, lifestyle and behavioral characteristics of these study 
participants based on olive oil consumption at baseline (no use vs. daily use). Overall, compared to participants reporting no olive oil use, those who reported daily olive oil consumption at baseline were younger, less likely to be smokers, and had lower BMI values; but had higher WHR and waist circumference, higher fasting levels of plasma lipids and were also more likely to have MetS at baseline (Table 1; all p-values $<0.05)$. Participants who consumed olive oil on a daily basis exhibited closer adherence to the Mediterranean diet, as indicated by the higher MedDietScore $(p=0.03)$, and a higher MUFA/SFA ratio intake $(p=0.006)$, whilst they also had lower energy intake from SFA ( $\mathrm{p}=0.007$ ) compared to participants reporting no olive oil use

(Table 1). As far as omega-3 intake is concerned, participants reporting olive oil use on a daily basis had higher levels of omega-3 fatty (g/d) acids compared to olive oil abstainers (mean \pm SD: $2.19 \pm 0.80$ vs. $2.11 \pm 0.59$, p-value $=0.002$ ).

\subsection{0-year CVD incidence and baseline olive oil consumption}

The 10-year fatal or non-fatal CVD event rate documented in the study was 157 cases/1000 participants [i.e., $\mathrm{n}=317$ subjects; men: n=198 (195 cases/1000 participants); women: n=119 (118 cases/1000 participants); p for gender difference $<0.001]$

A significant association was observed when compared participants with no olive use with those reported (mixed or exclusive) use as regards 10-year CVD risk (Table 1). Moreover, after classifying the participants into three groups, i.e. no olive oil use group, mixed use group and exclusive olive oil use, participants in the no olive oil use or in the mixed use group (i.e., consuming olive oil along with other dietary fats/oils) were 4.2-times and 5.3-times more likely to develop a CVD event during the 10-year study follow-up compared to those reporting exclusive olive oil use. 
The aforementioned comparisons may be prone to residual confounding due to various factors. Thus, multi-adjusted analysis was also performed, controlling for multiple CVD-related covariates (Table 2). Age-sex analysis revealed a significant protective association between exclusive olive oil consumption and the 10-year CVD incidence (Table 2, Model 1); even when other variables (i.e., BMI, smoking, physical activity status, education, hypertension, diabetes, hypercholesterolemia, and MetS) were entered in this model as potential confounders or mediating factors, participants who consumed exclusively olive oil at baseline had a 93\% lower risk of a CVD event during the study follow-up period compared to the no olive oil use group (Table 2, Model 2). Similarly, when MedDietScore and omega-3 fatty acids intake were entered in the model, the effect size of the association between olive oil use and CVD risk remained unaltered (data not shown). Moreover, no significant interactions were observed between olive oil use and the aforementioned potential confounders / mediating factors.

Taking into account that sub-clinical, low-grade inflammation could be a possible mediator of the association between exclusive olive oil consumption and the CVD risk, selected relevant circulating biomarkers were entered, consecutively (and separately) in the fully adjusted model (Model 2). Amongst these tested models, only adjustment for fibrinogen plasma levels showed a significant mediation of olive oil use on CVD risk (Model 3, Table 2; p for Sobel's test 0.05). Contrary, the models including CRP, ox-LDL, IL-6 and homocysteine, did not alter the impact of olive oil use on CVD risk (OR=1.05, 95\% CI: 0.98-1.12; OR=1.00, 95\% CI: 0.99-1.01; $\mathrm{OR}=1.02, \quad 95 \% \quad \mathrm{CI}: \quad 0.78-1.34 ; \quad \mathrm{OR}=1.01, \quad 95 \% \quad \mathrm{CI}: \quad 0.97-1.03, \quad$ respectively) (models/data not shown). 


\section{Discussion}

The present study reported novel data from a large cohort of prospectively followed adults without pre-existing CVD, showing a significant inverse association between exclusive use/consumption of olive oil and the 10-year CVD incidence, irrespective of the level of adherence to the Mediterranean diet and or other important constituents (e.g., omega-3 fatty acids intake). Of note, the reported results further suggest that fibrinogen, which is a key plasma coagulation factor with a potential proinflammatory role in vascular wall disease [20], may have a mediating effect in this protective association. Interestingly, there is evidence in the literature indicating that olive oil consumption can lower plasma fibrinogen levels and impact on platelet function, thrombogenesis and fibrinolysis [21, 22]. The fact that fibrinogen levels may better reflect vascular damage, could, partially be an explanation of the present study's findings, regarding the lack of mediating effect from the other inflammatory markers levels. However, the link between olive oil consumption, haemostasis, subclinical inflammation and CVD offers an intriguing hypothesis, which requires further experimental research. The presented findings are of particular importance for public health strategies on nutrition/diet and primary CVD prevention, supporting the notion that olive oil may be a key-player for the protection against CVD in the general population, potentially involving haemostasis and pro-inflammatory mechanisms.

The beneficial effects of olive oil are mainly attributed to its high content of MUFA (i.e., mainly to oleic acid), although other minor compounds/components, such as lipid molecules (e.g., squalene, tocopherols, triterpernic alcohols) and phenols (e.g., hydroxytyrosol and oleuropein) may also have important biological properties. This has particular importance, as recent studies have showed that hydroxytyrosol helps to improve plasma lipids levels and repair oxidative damage related to CVD [8, 
22]. Moreover, in a recent study located in southern Spain a greater dietary consumption of polyphenols in olive oil among participants who more closely followed the Mediterranean diet pattern, was observed; a fact that may also explain the cardioprotective effects of olive oil [23]. Of note, its high MUFA content confers to olive oil (virgin or refined) high resistance to elevated temperatures, thus allowing it to be reutilized more than once for frying [24]. In comparison to SFA from butter and margarine, olive oil/MUFA reduces low-density lipoprotein cholesterol (LDL) and favors an increase in high-density lipoprotein cholesterol (HDL) levels [8]. Furthermore, taking into consideration that the phenolic compounds of olive oil are potential scavengers of superoxide radicals and inhibit LDL oxidation, the effect of olive oil consumption on preserving the endothelial function may offer an additional benefit against the links between endothelial dysfunction, oxidative stress, inflammation and CVD [25, 26]. Moreover, a recent meta-analysis of randomized controlled trials (RCTs) investigating the effects of olive oil on markers of inflammation and endothelial function suggested that both pro-inflammatory biomarkers (CRP and IL-6) and endothelial function (flow-mediated dilatation) were favorably affected following interventions with olive oil [27]. It becomes evident, that all these mechanisms support the advantages of exclusive olive oil consumption compared to SFA-rich dietary patterns (e.g. cooking with butter and margarine) and may explain its protective role against CVD [8, 23].

It is also important to note the present findings are in accord with the existing epidemiological evidence from prospective studies showing that high olive oil consumption is inversely associated with CVD risk [10, 28, 29]. Indeed, in the EPICOR study reduced CVD risk (HR=0.56; 95\%CI: 0.31-0.99) was observed among Italian women in the highest quartile of olive oil consumption during the study 
follow-up period (approximately 8 years) [28]. Moreover, in the EPIC-Spain study (13.4 years of follow-up) participants in the highest quartile of olive oil consumption had a 44\% lower risk in CVD mortality compared to those not consuming olive oil [10]. The beneficial impact of olive oil consumption against CVD was further supported by the PREDIMED study, which showed that a Mediterranean diet supplemented with extra-virgin oil was associated with a clear reduction in CVD events (HR=0.70; 95\%CI: 0.54-0.92) in individuals at high CVD risk [29]. As such, the results presented are in agreement with the results of the PREDIMED study that highlighted olive oil consumption as a crucial Mediterranean Diet component for CVD prevention [11, 29]. It is of interest that the observed findings were not altered by the dietary pattern that the ATTICA study participants followed. Specifically, although olive oil consumers were higher adherers to the traditional dietary pattern and also reported higher omega-3 fatty acids intake, when adherence to the Mediterranean diet was taken into account in the epidemiological models, no alterations were observed in the tested relationship between olive oil and CVD risk, enhancing the important role of olive oil on the cardiovascular system.

Taking into consideration the traditional dietary patterns in Europe, it is not surprising that most of the limited existing prospective studies on the association between exclusive olive oil use and CVD risk are from Greece [12, 30, 31]. Although these previous studies focused on patients with coronary artery disease, their results also agree with the findings of the present study [12, 30, 31]. Indeed, the Greek CARDIO2000 case-control study, examined the association between exclusive olive oil consumption and the risk of developing acute coronary syndromes (ACS) in a large sample of ACS patients and CVD-free controls (748 cases and 1048 controls) showing a protective effect for the exclusive olive oil use against ACS (OR=0.53, 
95\% CI: 0.34-0.71) [12]. Moreover, the prospective GREECS study for secondary CVD prevention among patients with a history of ACS, exhibited an association between exclusive olive oil consumption and lower risk of recurrent ACS events [31].

\subsection{Study strengths and limitations}

The data of this work were obtained through a reliable and prospective followup process in a well-characterized, large CVD-free cohort from the general population in which multiple CVD-associated risk factors were comprehensively assessed with standardized procedures/methods. The characteristics of the study design/protocol and population strengthen the significance of the findings, since the associations of the olive oil consumption pattern with long-term CVD-risk were explored at baseline in a unique cohort from a Mediterranean country that traditionally has the highest daily use of olive oil in food cooking/preparation, while adjusting for multiple confounders.

However, certain study limitations must be also acknowledged. As such, it should be noted that the baseline study examination was performed once, hence may be susceptible to measurement error. Furthermore, the daily quantity of olive oil consumption was not evaluated, and therefore, it was not able to provide information about the contribution of olive oil to the total fat intake, as well as fat from ready to eat meals. Moreover, there was no characterization of the different varieties of olive oil (e.g. common, virgin or extra virgin olive oil), and, thus, the role of polyphenol intake was not specifically explored in this context. Moreover, it is possible that a number of participants may have changed their olive oil use habits during the followup period. Nevertheless, the applied methodology is similar to that followed in other prospective epidemiological studies in this field and, thus, the results can be considered reliable and generally comparable.

\subsection{Conclusions}


The present study offers evidence revealing an independent inverse association between exclusive olive oil use/consumption and CVD incidence in a cohort of Caucasian/Mediterranean adults without previous CVD. In addition, the multi-adjusted analyses suggest that fibrinogen may play a mediating role in this protective association. As such, these findings from the ATTICA study cohort extend the existing epidemiological evidence in this field and highlight the beneficial impact of olive oil consumption, a key component of the Mediterranean diet, on primary CVD prevention.

\section{Acknowledgements}

The authors would like to thank the ATTICA study group of investigators: Yannis Skoumas, Natasa Katinioti, Labros Papadimitriou, Constantina Masoura, Spiros Vellas, Yannis Lentzas, Manolis Kambaxis, Konstanitna Paliou, Vassiliki Metaxa, Agathi Ntzouvani, Dimitris Mpougatas, Nikolaos Skourlis, Christina Papanikolaou, Aikaterini Kalogeropoulou, Evangelia Pitaraki, Alexandros Laskaris, Mihail Hatzigeorgiou and Athanasios Grekas, Eleni Kokkou for either assistance in the initial physical examination and follow-up evaluation, Efi Tsetsekou for her assistance in psychological evaluation and follow-up evaluation, as well as laboratory team: Carmen Vassiliadou and George Dedousis (genetic analysis), Marina ToutouzaGiotsa, Constantina Tselika and Sia Poulopouloou (biochemical analysis) and Maria Toutouza for the database management.

\section{Conflict of interest}

The authors declare that they have no conflict of interest.

\section{Contributors}


GMK had the concept of the paper, performed data analyses and interpreted the results; DP supervised the study and reviewed the paper, CC, EG CP, designed the study, critically reviewed the paper, EM, IK, CT, DT critically reviewed the paper. All authors approved the final version.

Funding: D.B. Panagiotakos and E. Georgousopoulou received research grants by Coca-Cola SA. The ATTICA Study has been funded by research grants from the Hellenic Cardiology Society and the Hellenic Atherosclerosis Society. 


\section{References}

1. European Cardiovascular Disease Statistics (2017) Web site. http://www.ehnheart.org/cvd-statistics.html. Accessed 17 July 2017

2. World Health Organization (2017) Cardiovascular Diseases Fact Sheet Web Site. WHO. http://www.who.int/mediacentre/factsheets/fs317/en/. Accessed 17 July 2017.

3. Trichopoulou A, Kouris-Blazos A, Wahlqvist ML, Gnardellis C, Lagiou P, Polychronopoulos E, Vassilakou T, Lipworth L, Trichopoulos D (1995) Diet and overall survival in elderly people. BMJ 311:1457-60.

4. Willett WC (2006) The Mediterranean diet: science and practice. Public Health Nutr 9:105-10.

5. Keys A, Menotti A, Karvonen MJ, et al. (1986) The diet and 15-year death rate in the seven countries study. Am J Epidemiol 124:903-15.

6. Psaltopoulou T, Sergentanis TN, Panagiotakos DB, Sergentanis IN, Kosti R, Scarmeas N (2013) Mediterranean diet, stroke, cognitive impairment, and depression: A meta-analysis. Ann Neurol 74:580-91.

7. Kastorini CM, Milionis HJ, Esposito K, Giugliano D, Goudevenos JA, Panagiotakos DB (2011) The effect of Mediterranean diet on metabolic syndrome and its components: a meta-analysis of 50 studies and 534,906 individuals. J Am Coll Cardiol 57:1299-313.

8. Covas MI, de la Torre R, Fitó M (2015) Virgin olive oil: a key food for cardiovascular risk protection. Br J Nutr 113 Suppl 2:S19-28.

9. Schwingshackl L, Hoffmann G (2014) Monounsaturated fatty acids, olive oil and health status: a systematic review and meta-analysis of cohort studies. Lipids Health Dis 13:154. 
10. Buckland G, Mayén AL, Agudo A, Travier N, Navarro C, Huerta JM, Chirlaque MD, Barricarte A, Ardanaz E, Moreno-Iribas C, Marin P, Quirós JR, Redondo ML, Amiano P, Dorronsoro M, Arriola L, Molina E, Sanchez MJ, Gonzalez CA (2012) Olive oil intake and mortality within the Spanish population (EPIC-Spain). Am J Clin Nutr 96:142-9.

11. Guasch-Ferré M, Hu FB, Martínez-González MA, Fitó M, Bulló M, Estruch R, Ros E, Corella D, Recondo J, Gómez-Gracia E, Fiol M, Lapetra J, Serra-Majem L, Muñoz MA, Pintó X, Lamuela-Raventós RM, Basora J, Buil-Cosiales P, Sorlí JV, Ruiz-Gutiérrez V, Martínez JA, Salas-Salvadó J (2014) Olive oil intake and risk of cardiovascular disease and mortality in the PREDIMED Study. BMC Med $12: 78$.

12. Kontogianni MD, Panagiotakos DB, Chrysohoou C, Pitsavos C, Zampelas A, Stefanadis C (2007) The impact of olive oil consumption pattern on the risk of acute coronary syndromes: The CARDIO2000 case-control study. Clin Cardiol 30:125-9.

13. Pitsavos C, Panagiotakos DB, Chrysohoou C, Stefanadis C (2003) Epidemiology of cardiovascular risk factors in Greece: aims, design and baseline characteristics of the ATTICA study. BMC Public Health 3:32.

14. Katsouyanni K, Rimm EB, Gnardellis C, Trichopoulos D, Polychronopoulos E, Trichopoulou A (1997) Reproducibility and relative validity of an extensive semiquantitative food frequency questionnaire using dietary records and biochemical markers among Greek schoolteachers. Int J Epidemiol 26: S118-127.

15. Panagiotakos DB, Pitsavos C, Stefanadis C (2006) Dietary patterns: a Mediterranean diet score and its relation to clinical and biological markers of cardiovascular disease risk. Nutr Metab Cardiovasc Dis 16: 559-568. 
16. Papathanasiou G, Georgoudis G, Papandreou M, Spyropoulos P, Georgakopoulos D, Kalfakakou V, Evangelou A (2009) Reliability measures of the short International Physical Activity Questionnaire (IPAQ) in Greek young adults. Hellenic J Cardiol 50:283-294.

17. American Diabetes Association Report of the Expert Committee on the Diagnosis and Classification of Diabetes Mellitus (2014) Diabetes Care 20:1183-97.

18. Grundy SM, Brewer HB Jr., Cleeman JI, Smith SC Jr., Lenfant C (2004) Definition of metabolic syndrome: report of the National Heart, Lung, and Blood Institute/American Heart Association conference on scientific issues related to definition. Circulation 109:433- 8.

19. Panagiotakos DB, Georgousopoulou EN, Pitsavos C, Chrysohoou C, Metaxa V, Georgiopoulos GA, Kalogeropoulou K, Tousoulis D, Stefanadis C; ATTICA Study group (2014) Ten-year (2002-2012) cardiovascular disease incidence and all-cause mortality, in urban Greek population: the ATTICA Study. Int J Cardiol $180: 178-84$

20. Davalos D, Akassoglou K (2012) Fibrinogen as a key regulator of inflammation in disease. Semin Immunopathol 34:43-62.

21. Oosthuizen W, Vorster HH, Jerling JC, Barnard HC, Smuts CM, Silvis N, Kruger A, Venter CS (1994) Both fish oil and olive oil lowered plasma fibrinogen in women with high baseline fibrinogen levels. Thromb Haemost 72:557-62.

22. Delgado-Lista J, Garcia-Rios A, Perez-Martinez P, Lopez-Miranda J, PerezJimenez F (2011) Olive oil and haemostasis: platelet function, thrombogenesis and fibrinolysis. Curr Pharm Des 17:778-85.

23. de la Torre-Robles A, Rivas A, Lorenzo-Tovar ML, Monteagudo C, MariscalArcas M, Olea-Serrano F. Estimation of the intake of phenol compounds from 
virgin olive oil of a population from southern Spain. (2014) Food Addit Contam Part A Chem Anal Control Expo Risk Assess. 31:1460-9.

24. Casal S, Malheiro R, Sendas A, Oliveira BP, Pereira JA. (2010) Olive oil stability under deep-frying conditions. Food Chem Toxicol 48:2972-9.

25. Ruiz-Canela M, Martínez-González MA (2011) Olive oil in the primary prevention of cardiovascular disease. Maturitas 68:245-50.

26. Schwingshackl L, Hoffmann G (2014) Monounsaturated fatty acids, olive oil and health status: a systematic review and meta-analysis of cohort studies. Lipids Health Dis 13:154.

27. Schwingshackl L, Christoph M, Hoffmann G (2015) Effects of Olive Oil on Markers of Inflammation and Endothelial Function-A Systematic Review and Meta-Analysis. Nutrients 7:7651-75.

28. Bendinelli B, Masala G, Saieva C, Salvini S, Calonico C, Sacerdote C, Agnoli C, Grioni S, Frasca G, Mattiello A, Chiodini P, Tumino R, Vineis P, Palli D, Panico S (2011) Fruit, vegetables, and olive oil and risk of coronary heart disease in Italian women: the EPICOR Study. Am J Clin Nutr 93:275-83.

29. Estruch R, Ros E, Salas-Salvadó J, Covas MI, Corella D, Arós F, Gómez-Gracia E, Ruiz-Gutiérrez V, Fiol M, Lapetra J, Lamuela-Raventos RM, Serra-Majem L, Pintó X, Basora J, Muñoz MA, Sorlí JV, Martínez JA, Martínez-González MA; PREDIMED Study Investigators (2013) Primary prevention of cardiovascular disease with a Mediterranean diet. N Engl J Med 368:1279-90.

30. Dimitriou M, Rallidis LS, Theodoraki EV, Kalafati IP, Kolovou G, Dedoussis GV (2016) Exclusive olive oil consumption has a protective effect on coronary artery disease; overview of the THISEAS study. Public Health Nutr 19:1081-7. 
31. Kouvari M, Notara V, Panagiotakos DB, Michalopoulou M, Vassileiou N, Papataxiarchis E, Tzanoglou D, Mantas Y, Kogias Y, Stravopodis P, Papanagnou G, Zombolos S, Pitsavos C; GREECS Study Investigators (2016) Exclusive olive oil consumption and 10-year (2004-2014) acute coronary syndrome incidence among cardiac patients: the GREECS observational study. J Hum Nutr Diet 29:354-62. 
Table 1. Selected characteristics (demographic, lifestyle, clinical and diet characteristics) of the ATTICA Study participants as regards olive oil use at baseline.

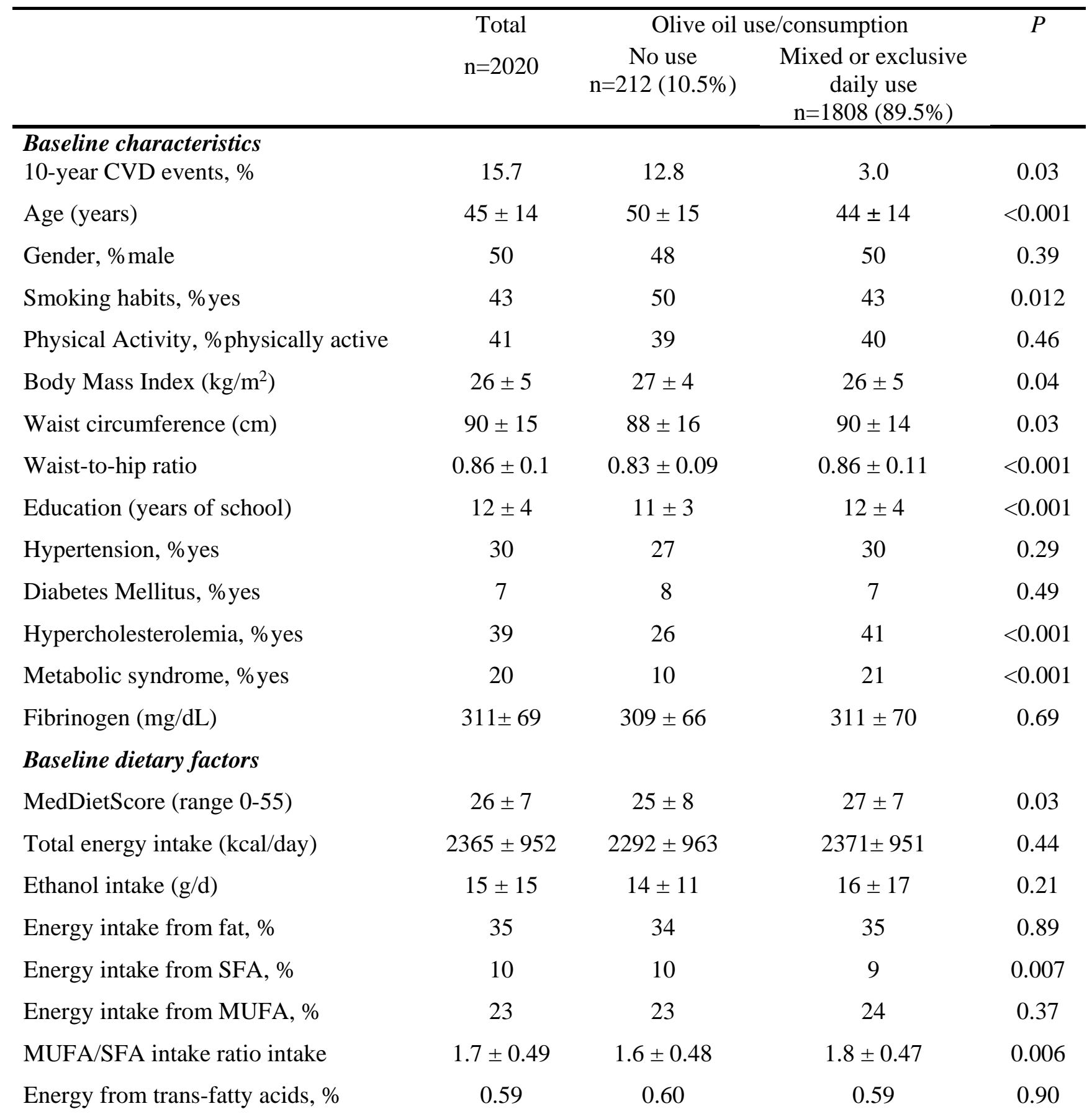

Continuous variables are presented as mean \pm standard deviation and categorical variables as frequencies. P-values for the comparisons between the no olive oil consumption group and the daily olive oil consumption group derived using the t-test, while for the comparisons of categorical variables using the chi-square test. MedDietScore: score evaluating the adherence to the Mediterranean diet (higher score values indicate better adherence); MUFA: mono-unsaturated fatty acids; SFA: saturated fatty acids 
Table 2. Results from the Cox proportional hazards models [Hazard Ratios (HR) and 95\% confidence intervals (CI)] that were applied to evaluate the 10-year risk of developing a fatal or non-fatal cardiovascular disease (CVD) event (outcome) according to the olive oil use/consumption pattern at baseline.

\begin{tabular}{|c|c|c|c|c|c|c|}
\hline & \multicolumn{6}{|c|}{ Hazard Ratios $^{1}$, 95\% Confidence Intervals } \\
\hline & \multicolumn{2}{|c|}{ Model 1} & \multicolumn{2}{|c|}{ Model 2} & \multicolumn{2}{|c|}{ Model 3} \\
\hline \multicolumn{7}{|l|}{ Olive oil use } \\
\hline No use (reference category) & 1.00 & - & 1.00 & - & 1.00 & - \\
\hline Mixed use with other oils/fats in cooking & 0.98 & $0.52,1.85^{2}$ & 0.91 & $0.45,1.83^{2}$ & 2.02 & $0.69-5.90^{2}$ \\
\hline Exclusive use in daily cooking & 0.11 & $0.01,0.95^{2}$ & 0.07 & $0.01-0.66^{2}$ & 0.24 & $0.02-2.54^{2}$ \\
\hline Age (per 1 year) & 1.09 & $1.08,1.10$ & 1.08 & $1.07-1.10$ & 1.07 & 1.06-1.09 \\
\hline Men vs. Women & 1.99 & $1.52,2.63$ & 1.72 & $1.28-2.33$ & 1.78 & $1.27-2.50$ \\
\hline Body Mass Index (per $1 \mathrm{~kg} / \mathrm{m}^{2}$ ) & - & - & 1.03 & $0.99-1.07$ & 1.03 & 0.99-1.07 \\
\hline Smoking habits (ever vs. never) & - & - & 1.30 & $0.96-1.78$ & 1.44 & $1.02-2.05$ \\
\hline Physically active vs. inactive & - & - & 0.97 & $0.72-1.30$ & 0.98 & $0.70-1.37$ \\
\hline Education ( $>14$ years vs. $<9$ years) & - & - & 0.97 & $0.93-1.01$ & 0.96 & $0.92-1.00$ \\
\hline \multicolumn{7}{|l|}{ History of } \\
\hline Hypertension $(y / n)$ & - & - & 1.04 & $0.77-1.42$ & 1.01 & $0.71-1.43$ \\
\hline Hypercholesterolemia $(y / n)$ & - & - & 1.07 & $0.80-1.43$ & 1.11 & $0.80-1.54$ \\
\hline Diabetes mellitus $(y / n)$ & & & 1.67 & $1.08-2.59$ & 1.47 & $0.89-2.44$ \\
\hline Metabolic syndrome (y/n) & - & - & 1.56 & $1.12-2.20$ & 1.76 & $1.19-2.60$ \\
\hline Fibrinogen (per 1 mg/dL) & & & & & 1.00 & $1.00-1.01$ \\
\hline
\end{tabular}

${ }^{1}$ Hazard Ratios derived from semiparametric Cox proportional hazards models.

${ }^{2} p$-value $<0.05$ 